\title{
STUDY OF OIL SPILL IN NORWEGIAN AREA USING DECOMPOSITION TECHNIQUES ON RISAT-1 HYBRID POLARIMETRIC DATA
}

\author{
P.V. Jayasri*, H.S.V. Usha Sundari, E.V.S. Sita Kumari, AVV Prasad \\ National Remote Sensing Centre, Indian Space Research Organisation, Hyderabad, India. \\ jayasri_pv@nrsc.gov.in*, ushasundari_hsv@nrsc.gov.in, sitakumari_evs@nrsc.gov.in, prasad_avv@nrsc.gov.in
}

Commission VIII, WG VIII/9

KEYWORDS: Oil spill, RISAT-1, hybrid polarization, decomposition, Stokes vector, SAR, look-alikes

\begin{abstract}
:
Over past few years Synthetic Aperture Radar(SAR) has received a considerable attention for monitoring and detection of oil spill due to its unique capabilities to provide wide-area surveillance and day and night measurements, almost independently from atmospheric conditions. The critical part of the oil spill detection is to distinguish oil spills from other natural phenomena. Stokes vector analysis of the image data is studied to estimate the polarized circular and linear components of the backscatter signal which essentially utilize the degree of polarization $(\mathrm{m})$ and relative phase $(\delta)$ of the target. In a controlled oil spill experiment conducted at Norwegian bay during $17^{\text {th }}$ to $22^{\text {nd }}$ June 2014 , RISAT-1 hybrid polarimetry images were utilized to study the characteristics of oil spill in the sea. The preliminary results obtained by using polarimetric decomposition technique on hybrid polarimetric data to decipher the polarimetric characteristics of oil spills from natural waters are discussed in the paper.
\end{abstract}

\section{INTRODUCTION}

To improve environmental protection and public safety, maritime surveillance is of major environmental and economical interest in the present scenario. Marine oil spill causes ecological pollutions that result in serious impacts to the quality of marine eco-environment because of major disasters akin to oil-tankers sinking and chronic events such as frequent illegal ship discharges and partly from large ship and oil-rig accidents. The study on oil spill aids in understanding its affect on biological and chemical cycles in the oceans and their influence on cryospheric and terrestrial changes (A.K. Fukuyama et al., 1998). The oil spill detectability essentially depends on wind speed, sea state and age of spills and the availability of additional information from other sources. Oil slicks are visible only for a limited range of wind speeds.

In view of dramatic short and long term effects caused by sea oil spills, as a matter of fact, the support of satellite -based remote sensing systems have been strongly encouraged. The field of oil slick remote sensing covers the science, technologies and operational services. Synthetic Aperture Radar (SAR) system provide a viable solution of oil slick monitoring. SAR intensity images are sensitive to surface roughness which is altered in the case of an oil spill. The scattering of open sea i.e., oil-free ocean surface is dominated by Bragg scattering (Bragg, 1913). After the oil spills, oil slicks spread to the sea surface dampen the Bragg waves and reduces radar backscattering coefficients resulting in a dark formation on SAR images. Radar backscatter values from oil spills are very similar to backscatter values from very calm sea areas and other ocean phenomena named "look-alikes" (e.g. ocean currents, eddies, biogenic films). There are different mechanisms responsible for the sea surface radar backscattering, which strongly depend on the incidence angle of the radar sensor. The SAR capacity to detect the oil spills on the sea surface is presented in terms of the local sea state conditions as well as technical characteristics of the sensor configuration (C Brown et al., 2003). Literature survey reveals that radar returns from steep and rough non-linear features of the sea surfaces associated with breaking of waves in the wide range of wavelength significantly contribute to back scatter coefficient even at low wind speed conditions. So exploiting the additional information in multi-polarization SAR measurements would be a logical step forward aiming at identifying and characterizing natural seepage slicks. (Gade et al., 1998) did some experiments to investigate whether space borne L-, C- and X-band multi-polarization SARs are capable of discriminating between films of different chemical properties, and found that discrimination is only possible at low and moderate wind speeds.

Maritime surveillance and monitoring using single linear dual polarization SAR modes have been studied extensively using ERS-1/2, RADARSAT-1 and ENVISAT ASAR data indicating very promising results for oil spill detection (M.F. Fingas, et al., 1997), (C. Liu et al., 2004) and (R. Touzi et al., 2004). SAR polarimetry can be made more reliable for maritime applications with the advent of new full-polarimetric satellite SAR systems such as the German TerraSAR-X, launched in June 2007, the Canadian RADARSAT-2, launched in December 2007, and the Japanese ALOS-PALSAR, launched in January 2006. Additionally, the future launches of the European Sentinel series and the Canadian RADARSAT Constellation Mission (RCM) with a noteworthy interest in maritime surveillance, will mark a fresh era for the operational use of SAR data. Moreover an increased activity is expected in the Arctic regions from the international maritime industry and oil/gas sector in the coming years. Novel knowledge and development of technology for oil spill remote sensing especially with compact/hybrid polarimetry in Arctic conditions are necessary to be explored by addressing the increased risk.

In order to comprehend the nature of monitoring and detection of oil spill in operational scenario, collecting the data obtained from controlled experiments is highly enviable so as to develop techniques for discrimination of oil types and slick thickness estimation. But, it is very difficult to get a permit to release oil into the sea to simulate accidental or illegal pollution. 
Nevertheless, Norwegian Clean Seas Association for Operating Companies (NOFO), oil industry and satellite service providers have shown interest in remote sensing research and technique development over the last few years. Release of various oil types, data collection and participation in annual large-scale oilon-water exercises in the Norwegian sea have taken place (Norway 2014). KSAT participate every year in this event, and use the opportunity to test out different satellite modes. As per one of the proposed oil-on-water exercises by NOFO, $50 \mathrm{~m}^{3}$ oil emulsion (testing high capacity boom - MOS sweeper) at 06:00 UTC and $40 \mathrm{~m}^{3}$ oil emulsion experiment at 12:00 are poured in Norwegian bay on 17 June 2014 as depicted in figure 1.

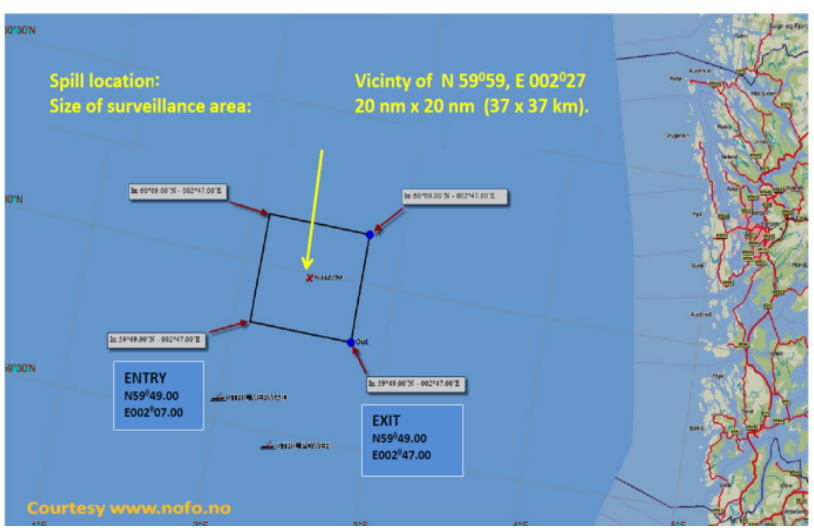

Figure 1. Oil-on-water experiment area at Norwegian Bay

This paper is organized as follows: a description of the theory at basis of hybrid polarimetry, stokes parameters, polarimetric decomposition and polarimetric capabilities of Radar Imaging Satellite (RISAT-1) are provided in section 2. Detailed discussion on the obtained results are illustrated in section 3 . The conclusions along with the future scope of the work are presented in section 4 .

\section{HYBRID POLARIZATION AND ITS DECOMPOSITION METHODOLOGY}

In most of applications, the main objective is to enhance the measurement potential of a Space-borne Synthetic Aperture Radar (SAR) in response to backscattered energy from a randomly distributed targets having unknown orientation relative to the transmitted radar signal.

\subsection{Background}

Polarimetric SAR systems have opened the doors to many innovative and new applications. The concept of quadraturepolarization (or full polarization) was introduced to remote sensing community in 1980s. Several recent papers (J.C. Souyris et al., 2005, Shane Robert Cloudie et al., 1996 and Micheal et al., 2009) have demonstrated that dual polarization SAR systems can, in certain situations, reproduce fully polarimetric information based on a few simple assumptions. Souyris et al. introduced the $\pi / 4$ compact polarimetric mode and in another study, Stacy et al. demonstrated that a compact polarimetric mode based on a circular polarization transmit and $(\mathrm{L}, \mathrm{R})$ circular polarization receive could be implemented using slightly modified form of the original compact polarimetry algorithm. It should be stressed at the outset that the hybridpolarization architecture (F.J. Charbonneau et al., 2010), or any other partial polarimetry scheme for that matter, is no substitute for quadrature polarization. Partial polarimetry is a major and efficient step up from a single channel system towards full polarimetric measurement capabilities. Partial polarimetry is a reasonable strategy when the system resources (power, mass, data volume or cost) preclude full polarimetry. With recent advances in polarimetry, SAR with Hybrid-polarity (CL-pol) architecture transmitting circular polarization $(\mathrm{H} \pm \mathrm{iV})$ and receiving two orthogonal mutually coherent polarizations, which is one demonstration of compact polarimetry enabling larger swath coverage, reduced PRF and SAR system complexity as compared to fully polarimetric systems. Among the three compact polarimetry modes, the Hybrid mode has been ranked to be the most promising in terms of performance and receiver complexity. The hybrid polarimetric data provides more information compared to linear single/dual polarimetric data for analyzing the behaviour of the various classes of targets. Moreover, circular polarimetry transmission is insensitive to Faraday rotation and has minimum sensitivity to cross talks between the channels. There are at least four Earth observation present and future satellite missions that are providing a compact polarimetry mode such as RISAT-1 (India), Sentinel-1, ALOS-2 (Japan), NISAR (NASA/ISRO L \& $\mathrm{S}$ band SAR), SAO-COM-1(Argentina), and Radarsat Constellation Mission (RCM, Canada).

\subsection{RISAT-1}

The first Hybrid polarimetry Space-borne SAR in Earth Observation orbit is India's Radar Imaging Satellite (RISAT-1) launched on 26th April, 2012 is a new-fangled gateway to remote sensing user community for land and oceanic applications. In on-board architecture of RISAT-1, two separate Transmit/Receive Modules (TRM) are used for two polarizations. This uniqueness enabled incorporation of Circular polarization in RISAT-1. These changes has catapult RISAT-1 to become the world's first space borne SAR to have circular polarization for earth imaging. This capability has added an extra dimension to RISAT-1 Operation and extended the potential for its data product applications. Basic requirement for Hybrid polarimetry is to feed $\mathrm{V}$ and $\mathrm{H}$ signal simultaneously to antenna with $90^{\circ}$ phase difference as shown in figure 2 . However, transmit pulse width is reduced to half, i.e., from $20 \mu \mathrm{sec}$ to $10 \mu \mathrm{sec}$, in the circular polarization mode, so that net power requirement is maintained.

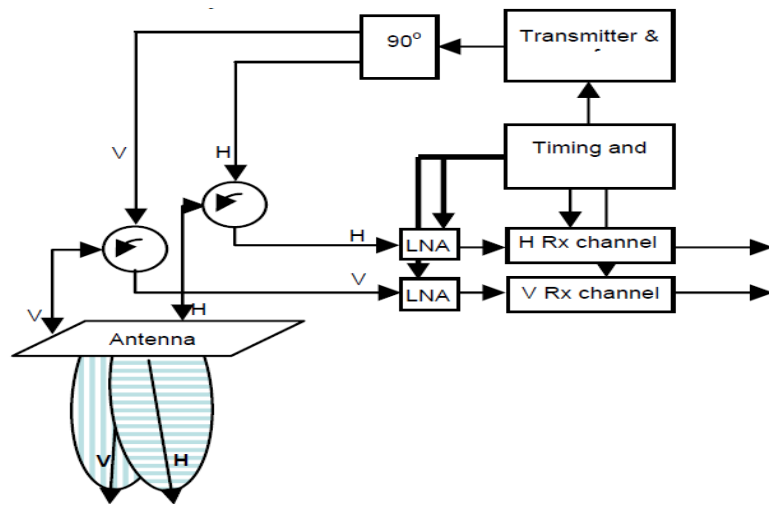

Figure 2. Hardware Realization of Hybrid Polarimetry

RISAT-1 operating in C-band $(5,35 \mathrm{GHz})$ has active phased array antenna technology supporting variety of resolutions and swath requirements (Tapan Misra et al., 2013). It is designed to provide constant swath for all beam elevation pointing and almost near constant Noise Equivalent Sigma Zero (NESZ). RISAT-1 supports single, dual, and Hybrid polarisations and acquires images in Stripmap (FRS-1), ScanSAR(MRS/CRS) and Spotlight modes(HRS) with resolutions and swaths as given in Table 1. 


\begin{tabular}{|l|l|l|l|}
\hline Imaging Mode & $\begin{array}{l}\text { Resolut } \\
\text {-ion }\end{array}$ & Swath & Polarization \\
\hline $\begin{array}{l}\text { Fine Resolution } \\
\text { SAR (FRS-1) }\end{array}$ & $3 \mathrm{~m}$ & $25 \mathrm{~km}$ & $\begin{array}{l}\text { Single, Dual, } \\
\text { Hybrid }\end{array}$ \\
\hline $\begin{array}{l}\text { Medium Resolution } \\
\text { SAR (MRS) }\end{array}$ & $25 \mathrm{~m}$ & $\begin{array}{l}115 \mathrm{k} \\
\mathrm{m}\end{array}$ & $\begin{array}{l}\text { Single, Dual, } \\
\text { Hybrid }\end{array}$ \\
\hline $\begin{array}{l}\text { Cours Resolution } \\
\text { SAR (CRS) }\end{array}$ & $50 \mathrm{~m}$ & $\begin{array}{l}223 \mathrm{k} \\
\mathrm{m}\end{array}$ & $\begin{array}{l}\text { Single, Dual, } \\
\text { Hybrid }\end{array}$ \\
\hline $\begin{array}{l}\text { Fine Resolution } \\
\text { SAR (FRS-2) }\end{array}$ & $9 \mathrm{~m}$ & $25 \mathrm{~km}$ & Quad pol \\
\hline $\begin{array}{l}\text { High Resolution } \\
\text { SAR (HRS) }\end{array}$ & $1 \mathrm{~m}$ & $10 \mathrm{~km}$ & $\begin{array}{l}\text { Single, Dual, } \\
\text { Hybrid }\end{array}$ \\
\hline
\end{tabular}

Table 1. Specifications of RISAT-1 Imaging modes

\subsection{Stokes Parameters}

The backscatter signal is usually partially polarized, that is, it contains a polarized component that has systematic polarization and an unpolarized component that has random polarization. The polarized component can be represented by a polarization ellipse, showing the path of the tip of the electric vector of the backscatter signal at a fixed point in space. The parameters of the polarization ellipse can be estimated from receive polarizations using the Stokes vector. The underlying Stokes parameter theory is well known (Stokes 1852 and Boerner et al., 1998). Stokes vector analysis of the image data will be used to estimate the polarized circular and linear components of the backscatter signal. Coherently dual-polarized received signals in the linear basis are sufficient to calculate the four Stokes parameters, which are rotationally invariant with respect to geometric trends in the scene since the transmit polarization is circular. Stokes parameters and their norms can be derived equally well for either linearly or circularly polarized backscatter generated in response to circularly polarized transmissions.

For radar like RISAT-1, the fundamental requirement in generating the Stokes parameters is that the complex amplitudes of the focused images are available from both orthogonally polarized received channels. The radar backscatter from the terrain includes fully and randomly polarized constituents primarily from volumetric materials that give rise to multiple internal reflections which obliterate the transmitted polarization characteristics. The polarized portion of the backscattering can be bifurcated into two classes namely single (odd) bounce (Bragg Scattering as well as specular reflection from a quasi planar surface perpendicular to transmitted signal) and double (even) bounce (dihedrals or di-planes) (R.K. Raney 2006). In response to a right-circularly polarized transmitted signal, circular polarimetry provides stokes parameters (S1, S2, S3, S4) by using data received in two mutually orthogonal channels. The four stokes parameters of the backscattered field are represented in matrix form as

$$
\left[\begin{array}{c}
S_{1} \\
S_{2} \\
S_{3} \\
S_{4}
\end{array}\right]=\left[\begin{array}{c}
\left\langle\left|E_{R H}\right|^{2}\right\rangle+\left\langle\left|E_{R V}\right|^{2}\right\rangle \\
\left\langle\left|E_{R H}\right|^{2}\right\rangle-\left\langle\left|E_{R V}\right|^{2}\right\rangle \\
2 \operatorname{Re}\left\langle E_{R H} E_{R V}{ }^{*}\right\rangle \\
-2 \operatorname{Im}\left\langle E_{R H} E_{R V}{ }^{*}\right\rangle
\end{array}\right]
$$

In each case, $E_{R H}$ represents complex voltage received by the channel with right-circular transmit and horizontal receive, $E_{R V}$ represents complex voltage received by the channel of rightcircular transmit and vertical polarization receive, $*$ indicates complex conjugate, $<\ldots>$ denotes ensemble average, and Re and $I m$ represent the real or the imaginary value (respectively) of the complex image.

\subsection{Hybrid Polarimetric Decomposition}

Polarimetric analysis in related fields has developed a mature alternative technique known as decomposition, in which two or more suitably selected parameters are used jointly to classify fundamental characteristics of the observed field. Based on the derived stokes parameters, several useful quantitative measures follow like circular polarization ratio (CPR, representative of scattering associated with dihedral reflection), Degree of linear polarisation $\left(\mathrm{m}_{\mathrm{L}}\right.$, an indicator of volume $\mathrm{Vs}$ subsurface scattering), Degree of Polarisation (DoP) (m, representative of polarized and diffused scattering) and relative phase $(\delta)$ between the two linear E-vectors of the backscattered field (an indicator of double bounce scattering). The state of polarization of an electromagnetic wave can be characterized by the DoP and relative phase expressed in terms of Stokes parameters as

$$
\begin{array}{cc}
m=\frac{\sqrt{S_{2}^{2}+S_{3}^{2}+S_{4}^{2}}}{S_{1}^{2}} & 0 \geq m \geq 1 \\
\delta=\tan ^{-1}\left(\frac{S_{4}}{S_{3}}\right) & -180^{\circ} \geq \delta \geq 180^{\circ}
\end{array}
$$

The parameter ' $m$ ' helps to determine the nature of the object based on the backscatter of the electromagnetic field and hence becomes a great interest for operational real-time detection as its estimation is computationally efficient. The surface of the unit Poincar'e sphere corresponds to $\mathrm{m}=1$, and represents all totally polarized states. Once polarized wave interacts with a random medium, the polarization state of the backscattered wave may change. Hence, depolarization is associated with a reduction in the polarization of incident states. A decomposition method along with suitable weighting functions has to be made available which maps Stokes parameters to RGB image space.

In response to circularly polarized illumination, multiple scattering within a subsurface volume in combination with surface scattering imposes linear polarization components into the backscatter. This effect can be observed through the four stokes parameters, but not through the detected images alone. The degree of linear polarization which is closely related to the observed field's entropy is one means of quantifying the effect. The decomposition techniques include $\mathrm{m}-\delta$ and $\mathrm{m}-\boldsymbol{\chi}$ decompositions based on elements of Stokes 'child' parameters $(\mathrm{m}, \delta$ and $\chi$ ) that best suits for hybrid circular polarimetric data are suggested in literature (R. K. Raney 2012). The power scattered by odd and even bounce targets are obtained by applying sinusoidal weighting function $(1-\sin (\delta)) / 2$ for even bounce targets and $(1+\sin (\delta)) / 2$ for odd bounce targets respectively. The voltage level contribution from odd, even and volumetric targets to generate scattering type RGB image using $\mathrm{m}-\delta$ decomposition are expressed as

$$
\begin{aligned}
& f_{\text {odd }}=\sqrt{S_{1} * m * \frac{1+\sin (\delta)}{2}} \\
& f_{\text {even }}=\sqrt{S_{1} * m * \frac{1-\sin (\delta)}{2}} \\
& f_{\text {Volume }}=\sqrt{S_{1} *(1-m)}
\end{aligned}
$$

It is noted that that in order to decipher the linearly polarization constituent in the transmitted field, Poincare' ellipticity parameter $\chi$ (chi) is the second decomposition variable which is 
one of the three principal components $(\mathrm{m}, \chi, \psi)$ that are necessary and sufficient to describe the polarized portion of a partially-polarized quasi-monochromatic EM filed of average strength $\mathrm{S}_{1}$.

$$
\begin{gathered}
f_{\text {odd }}=\sqrt{S_{1} * m * \frac{1+\sin (2 \chi)}{2}} \\
f_{\text {even }}=\sqrt{S_{1} * m * \frac{1+\sin (2 \chi)}{2}} \\
f_{\text {Volume }}=\sqrt{S_{1} *(1-m)}
\end{gathered}
$$

\section{RESULTS AND DISCUSSION}

This study is at preliminary stage, with the focus currently on understanding the characteristics and application of hybrid polarimetry decomposition techniques to detect the oil spill in oceans. This pilot study has been taken up based on few studies done by Salberg et al, and Shirvany et al on simulated hybrid polarization mode from full polarimetric data for oil slick detection.

\subsection{Datasets}

Our work is a theoretical investigation of the potential of hybrid polarimetry in discriminating the look-alikes from oil spills with the available data sets. For the present paper, RISAT-1 SingleLook Complex (SLC) SAR data acquired in circular Fine Resolution Stripmap (cFRS-1) mode having $75 \mathrm{MHz}$ chirp bandwidth acquired on 17th June 2014 at IST 17:38:03 which is approximately 2 hours before the $40 \mathrm{~m}^{3}$ oil emulsion experiment was considered for applying the polarimetric decomposition method which essentially utilize the degree of polarization, relative phase of the targets and ellipticity parameter of received polarization signal. To understand the look-alike features, multiple data sets corresponding to 20th June 2014 acquired in cFRS- 1 are considered. The datasets considered for the analysis are tabulated in Table 2. Literature survey reveals that radar returns from steep and rough non-linear features of the sea surfaces associated with breaking of waves in the wide range of wavelength significantly contribute to back scatter coefficient even at low wind speed conditions. A wind speed between $12 \mathrm{~m} / \mathrm{s}$ and $14 \mathrm{~m} / \mathrm{s}$ should possibly be considered as the upper limit for all space borne SAR imagery of oil spills ( Litovchenko et al., 1999). Due to the wind speed at the time of imaging and angle of incidence along with the time delay between oil-on-water exercise w.r.t imaging time, the data sets corresponding to orbit no. 744, 6203 resulted in more of lookalike features.

\begin{tabular}{|l|l|l|l|}
\hline Date of Pass & Orbit & $\begin{array}{l}\text { Incidence } \\
\text { angle }\end{array}$ & $\begin{array}{l}\text { Time of } \\
\text { imaging (IST) }\end{array}$ \\
\hline $14-06-2012$ & 744 & $41.023^{0}$ & $06: 03: 59.838$ \\
\hline $11-06-2013$ & 6203 & $38.943^{0}$ & $06: 12: 51.925$ \\
\hline $17-06-2014$ & 11835 & $49.440^{0}$ & $17: 38: 03.939$ \\
\hline $20-6-2014$ & 11843 & $24.519^{0}$ & $06: 22: 39.180$ \\
\hline
\end{tabular}

Table 2. RISAT-1 data sets considered for analysis

\subsection{Decomposition Results}

A novel characteristic m-delta, m-Chi polarimetric decomposition methods suggested by (Dr. K. Raney et al., 2011) are implemented along with signal processing techniques on hybrid polarimetric SAR data so as to map Stokes parameters to RGB image space to detect oil spill from natural waters as depicted in figure 3 . The yellow circles represents the $50 \mathrm{~m}^{3}$ oil emulsion poured approximately 14 hours before the image acquisition time and hence appearing as small dark

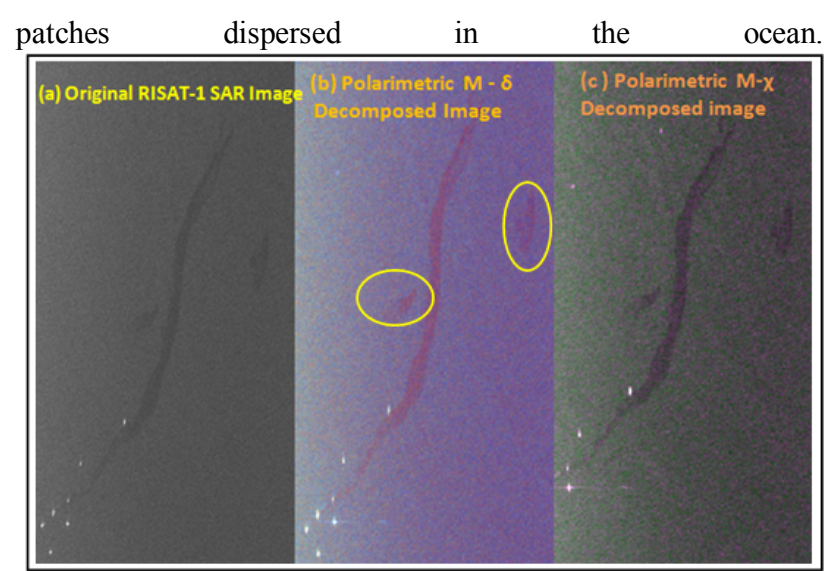

Figure 3. Original SAR image (a) along with RGB image constructed using derived Stokes parameters representing single, double and volumetric scattering mechanism using (b)m$\delta$ and (c)m- $\chi$ decomposition techniques. The long curve corresponds to the radar signature of $40 \mathrm{~m}^{3}$ oil emulsion.

After we generate the stokes parameters, the computation of degree of polarization $(\mathrm{m})$ helped as a significant distinguisher for characterizing the polarity of the backscatter. The mean value of $\mathrm{m}$ for oil spill area is $\sim 0.379$ as plotted in Figure 4 represent that the wave is depolarized. It signifies that there exists subtle volumetric type of scattering in the oil spill area as compared to clear water regions where mean ' $\mathrm{m}$ ' value is closer to 0 . The relative phase $(\delta)$ parameter derived from Stokes parameters is a sensitive indicator which distinguishes the difference between the surface and double bounce scattering when circularly polarized wave is transmitted. As the estimated $\delta$ has the mean positive value of $11.37^{0}$ in oil spill region indicating diffused scattering as compared to open sea water of $34.039^{0}$.

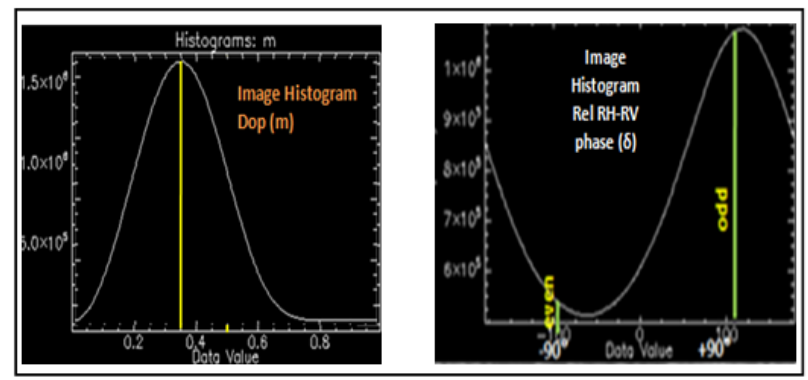

Figure 4. The distribution of degree of polarization and relative phase for oil spill regions.

The ellipticity parameter $(\chi)$ is a sensitive indicator of type of circular polarization transmitted and corresponds to a parameter on the Poincare'sphere.

$$
\sin 2 \chi=\left(\frac{s_{4}}{m s_{1}}\right) \quad-45^{0} \geq \chi \geq 45^{0}
$$

In general oil spill detection methodologies traditionally use arbitrary selected quantitative and qualitative statistical features for classifying dark objects on SAR images into oil spills or look-alike phenomena. As the data set considered is right circular transmitted signal, the estimated mean value of $\chi$ as shown in Figure 5 has a positive angle less than $+45^{0}$ for both oil slick and look -alike data sets. 

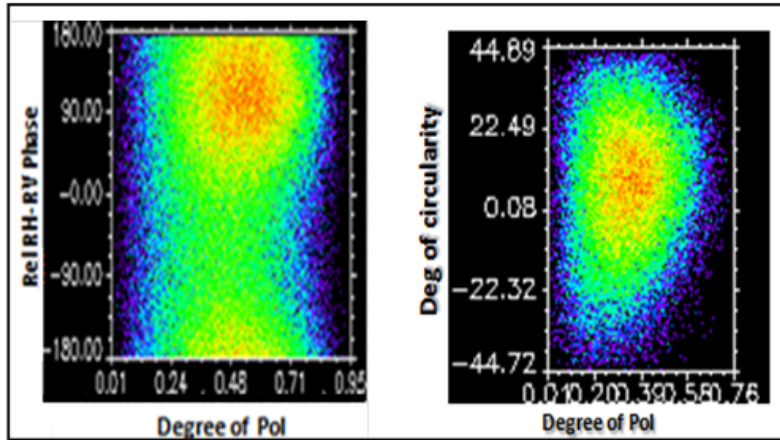

Figure 5. The scatter plots representing the distribution of degree of polarization verses relative phase (in left) signifying bragg scattering with $+90^{\circ}$ phase and degree of polarization verses Degree of circularity signifying the concentration of data values in positive angles ( in right).

The circular polarisation ratio (CPR) which relates to the surface roughness, which increases with increase in surface roughness. This quantity is equal to the magnitude of the same sense (i.e., right handedness of the transmitted circular polarization) divided by the opposite sense polarization signals that are received. Additionally, CPR is a supplementing information to aid the interpretation of the results. It is observed from the estimation of CPR that the mean value for oil is 1.01 as compared to 0.909 for open sea water. It reveals that surface roughness is quite less. The wind speed corresponding to this time is $\sim 4-5 \mathrm{~m} / \mathrm{s}$ (based on the estimations made from SARAL Altimeter data on 17th June 2014) with shallow incidence angle.

The degree of linear polarization $\left(\mu_{\mathbb{L}}\right)$ or coherency parameter for oil slick region, normal sea and look-alikes as shown in Figure 6 are observed to have close relation with degree of polarization and is given by

$$
\mu_{\mathbb{L}}=\frac{\sqrt{S_{2}^{2}+S_{3}^{2}}}{S_{1}}
$$

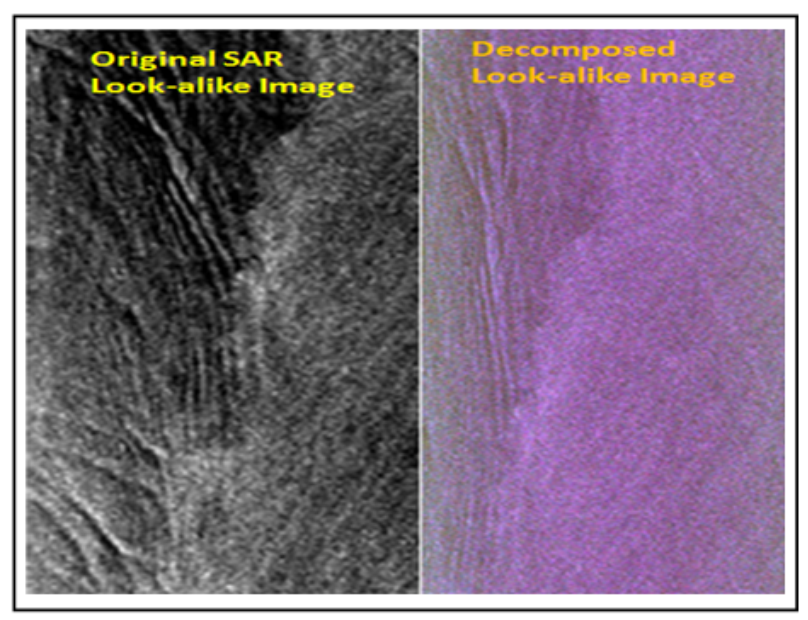

Figure 6. Original SAR look-alike image (left) along with RGB image constructed using derived Stokes parameters using $m-\chi$ decomposition technique (right).

A noise analysis has been performed on the bottom left of the image comparing the backscatter levels with the sensor noise floor. Selected region of around 75 by 75 pixels within the oil slick are selected for which mean sigma zero is computed. RISAT-1 SAR sensor has minimum sigma naught of $\sim-17 \mathrm{~dB}$. It is observed that the mean back scattering coefficient of oil spilled area and open sea waters are found to be $\sim 4.6 \mathrm{~dB}$ and $\sim 3.55 \mathrm{~dB}$ above the corresponding noise equivalent sigma naught (NESZ) in RV and RH channels respectively with more standard deviation. With the application of gamma filter to reduce the effect of speckle noise in Single look complex image, standard deviation in oil spill regions are found to have less than one standard deviation above the noise floor.

The application of $\mathrm{m}-\delta$ decomposition methodology and its validation has been evaluated by considering an image where trihedral corner reflectors were deployed having three perpendicular plates resulting in odd bounce effect is represented in blue colour (Jayasri et al., 2013). Even the RISAT-1 data has been acquired in Medium Resolution ScanSAR mode having large swath of $115 \mathrm{~km}$ with $25 \mathrm{~m}$ resolution in hybrid polarization on $18^{\text {th }}$ June 2014 i.e., one day after the experiment, the oil spill was dispersed and appeared as small dark patches at three regions in the middle of the scene in ground range product. This acquired data in ScanSAR mode is not utilized for analysis as Single Look Complex products in this imaging mode are declared to user community.

\section{CONCLUSIONS AND FUTURE SCOPE}

Scattering decomposition of polarimetric SAR data is essential to understand the predominant scattering type from a single or averaged resolution cell. Power scattered by even bounce and odd bounce targets are dependent on the state of polarization and relative phase values of the targets. If is found that some of the derived Stokes parameters like CPR, ' $m$ ', ' $\delta$ ' and ' $\chi$ ' are effective in characterizing radar scattering associated with oil spill and open water in terms of roughness of the surface and dominant scattering type in oceans. At the early stage of this experiment, shapes of oil slicks mainly appeared as patches. As time passed by, quite a few parts of oil slick appeared as distant spreads as its shape and position were affected by winds, ocean currents etc. Among the datasets considered over the experiment period, the effect of incidence angle (steeper incidence angle of $\sim 24.519^{\circ}$ ) and ocean currents tends to lookalikes in the images resembling oil spill. Moreover clear water depolarizes the incident wave more than oil for steep incidence angles, and less than oil for shallow incidence angles. The transition incidence angle suggested is around $40^{\circ}$. We also note that the distinction between types of slicks become more pronounced as the incidence angle increases. Encouraging preliminary results were obtained by using polarimetric decomposition technique on RISAT-1 hybrid pol data.

To the author's knowledge, there are very few existing publications on the behaviour of different types of oils with respect to open sea waters for hybrid/compact dual polarimetry satellite SAR data. Till now, the possibilities of using compact polarimetry for oil slick detection has been investigated by simulating compact pol from Radarsat-2 and TerraSAR -X quad-pol data. Significant C-band SAR measurements collected in compact polarization in large scale experiments with oil released on open water are required in order to conclude concretely the comprehensive use in detecting oil spills. However, our study reveals that a constructive work can be carried out over the coming years in establishing the procedures and algorithms in compact polarimetric decomposition techniques to assist the operators and ground personnel in determining the priority areas of oil slicks. It is possible to get at regular intervals similar data sets so that oil spill at rigs can be generated for any island or peninsular countries. The major issues to be addressed by the community in the near future 
cover research and algorithm development for compact polarimetry, oil slick drift modelling and its characterisation, and improved revisit time by novel polarimetric sensor technologies. In order to prepare better for future events, a mammoth effort should be made to uplift promising innovative research results to the operational scenario.

\section{ACKNOWLEDGEMENTS}

Authors are highly grateful to Dr. V.K. Dadhwal, Director, NRSC for the encouragement given throughout this paper. We are thankful to Sri Dharmendra Kumar Pandey, SAC/ISRO, Sri L.J. Vijay Kumar, MCF/ISRO and Dr. S.K. Sasmal of Oceangraphy, NRSC for their technical support. The authors would like to thank Dr. N. Aparna and Mrs. A.V. Ramani of NRSC Data Centre for their valuable information on RISAT-1 imaging plans on Norwegian area. We are thankful to the staff of MRSDPD/MRS\&GDP/NRSC for their kind cooperation in presenting this paper.

\section{REFERENCES}

A.K. Fukuyama, G. Shigenaka, G.R. VanBlaricom 1998. "Oil soil impacts and the biological basis for response guidance: an applied synthesis of research on tree subartic interidal communities, NOAA Tech, memorandum NOS ORCA 125, Seattle Washington.

Boerner, W-M., H. Mott, E. Luneburg, C. Livingstone, B Brisco, R.J. Brown and J.S. Paterson 1998, Polarimetry in remote sensing: basic and applied concepts, in principles and applications of imaging radar, edited by F.M. Henderson and A.J. Lewis, chap 5, pp:271-358, John Wiley, Hoboken N.J.

Bragg, W., 1913. The diffraction of short electromagnetic waves by a crystal. In: Proceedings of the Cambridge Philiosophical Society, Vol. 17, pp. 43-57.

C. Brown, M. Fingas, and R. Hawkins, 2003 "Synthetic aperture radar sensors: Viable for marine oil spill response," in Proc. 26th Arctic and Marine Oil Spill Program (AMOP) Tech. Seminar, Victoria, Canada,

C. Liu, P.W. vachon, and G.W. Geiling, 2004 "Improved ship detection using polarimetric SAR data", in Proc. IGARSS, Anchorage, AK, Sep 2004. Vol.3, pp.1800-1803, vol.3

F.J. Charbonneau, B. Brisco, R.K. Raney, H. McNairn, C. liu, P.W. Vachon, J. Shang, R. DeAbreu, C. Chmapagne, A. Merzouki and T. Geldsetzer 2010. "Compact polarimetry overview and applications assessment", Canadian Journal of remote sensing, vol.36, suppl.2, pp S298-S315.

Gade, M ., Alpers, W., Masuko, H., and Kobayashi, T., 1998 "Imaging of biogenic and anthropogenic ocean surface films by the multifrequency/multipolarization SIR-C/X-SAR," Journal of Geophysical Research, vol.103, no.C, PP. 18851-18866.

Jayasri P.V., Usha Sunadri HSV, Sita Kumari EVS, Prasad AVV 2013, M-delta decomposition of Hybrid Dual Polarimetric RISAT-1 SAR Data, 9th International Radar Symposium India(IRSI-2013).

J.C. Souyris, P. Imbo, R. Fjortoft, S. mingot and J.S. Lee 2005. " Compact polarimetry based on symmetry properties of geophysical media: the $\pi / 4$ mode", IEEE Trans. Geosci. Remote Sens., vol.43, no.3, pp.634-646.

Litovchenko. K, Ivanov A \& Ermakov S(1999). Detection of oil slicks parameters from ALMAZ-1 and ERS-1 SAR imagery. Proc. IGRASS'99, vol.3 (pp.1484-1486).

M.F. Fingas and C.E. Brown, 1997 "review of oil spill remote sensing”, Spill science Technology Bulletin vol.4, no.4, pp.199208,

Michael E. Nord, Thomas . Ainsworth, Jong-Sen Lee, Nick J.S. Stacy 2009. " Comparison of compact polarimetric synthetic aperture radar modes", IEEE transaction on geosciences and remote sensing, vol.47, no.1, January 2009.

Norwegian Clean Seas Association for Operating Companies, 2014 (nofo), www.nofo.no.

R.K. Raney, 2006. " Dual -polarized SAR and Stokes Parameters", IEEE Geoscience and remote sensing letters, VOL.3, No.3, July 2006.

Raney R.K. 2011, A perspective on compact polarimetry, IEEE Geosci. Remote sensing, Newsletter, vol.160, pp.12-18,sep 2011.

R.K. Raney, 2012 "Synthetic aperture radar hybrid-quadraturepolarity method and architecture for obtaining the stokes parameters of radar backscatter", US 8,258,996 B2, Sep 4, 2012.

R. Touzi, F.J. Charbonneau, R.K. kawkins and p.W. Vachon, 2004 "Ship detection and characterization using polarimetric SAR", Candian Journal of Remote Sensing, Vol.30, no.3, pp.552-559.

R. Touzi, "Compact-hybrid versus linear-dual and fully polarimetric SAR", Proc. of 4th International Workshop on Science and Applications of SAR polarimetry and polarimetric Interferometry- PolInSAR 2009', 26-30 January 2009, Frascati, Italy (ESA SP-668, April 2009).

Salberg, A.B, Rudjord O and Solberg A.H.S., 2012 oilspill detection in compact polarimetry SAR images, In proc. of SeaSAR 2012, Tromso, Norway, 18-22 June 2012.

Shane Robert Cloude, Eric Pottier, 1996. "A review of target decomposition theorem in radar polarimetry", IEEE transactions on Geoscience and remote sensing, Vol.34, No.2, March 1996.

Shirvany. R., Chabert. M., and Tourneret. J, 2012 " ship and oilspill detectionusing degree of polarimzation in linear and hybrid/compact Dual-pol SAR, IEEE Journal of selected topics in appliederath observations and remote sensing, vol.5, No.3.

Stokes G.G. 1852, On the composition and resolution of streams of polarized light from different sources. Trans Cambridge Philos. Soc., 9, 399-416.

Tapan Misra, S.S. Rana, N.M. Desai, D.B. Dave, Rajeevjyoti, R.K. Arora, C.V.N. Rao, B.V. Bakori, R. Neelakantan, J.G. Vachchani 2013, "Current Science", Vol 104, No.4, Feb 2013. 